\title{
Power and spectrum efficiencies of an optical satellite uplink with spatially diverse beams and incoherent combining
}

H. Ordouei, M. Krzyzek, N. Perlot, R. Freund

H. Ordouei, M. Krzyzek, N. Perlot, R. Freund, "Power and spectrum efficiencies of an optical satellite uplink with spatially diverse beams and incoherent combining," Proc. SPIE 11852, International Conference on Space Optics - ICSO 2020, 118524H (11 June 2021); doi: 10.1117/12.2599660

SPIE Event: International Conference on Space Optics - ICSO 2021, 2021, Online Only 


\section{International Conference on Space Optics-ICSO 2020}

Virtual Conference

30 March-2 April 2021

Edited by Bruno Cugny, Zoran Sodnik, and Nikos Karafolas
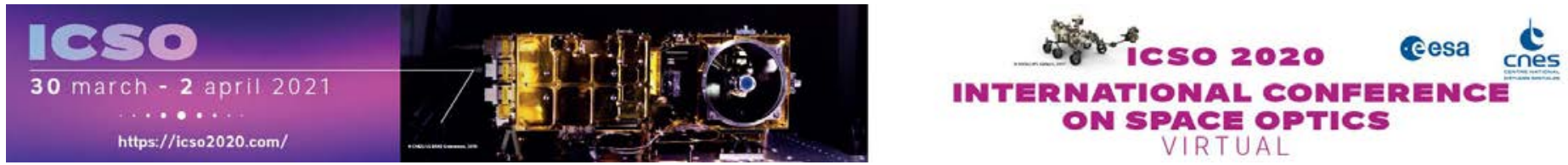

\section{Power and spectrum efficiencies of an optical satellite uplink with spatially diverse beams and incoherent combining}

\section{Cesa isoporecestings denes}




\title{
Power and spectrum efficiencies of an optical satellite uplink with spatially diverse beams and incoherent combining
}

\author{
H. Ordouei, M. Krzyzek, N. Perlot*, R. Freund \\ Fraunhofer Institute for Telecommunications, Heinrich Hertz Institute, Einsteinufer 37, 10587 Berlin \\ *nicolas.perlot@hhi.fraunhofer.de
}

\begin{abstract}
Existing radio-based satellite up- and downlinks are reaching their limits in terms of transmission rate. In order to enable higher data rates, optical links are being researched and developed. On ground, spatially displaced multi-aperture systems using different wavelengths can mitigate atmospheric scintillation and increase the uplink transmit power.

A four-aperture free-space optical communication system was demonstrated at Fraunhofer HHI over a bidirectional link. For the representative uplink, each array aperture sent a beam carrying the same data but over a different wavelength to avoid inter-beam interferences. At the receiver, the combined signals are optically amplified, optically filtered, incoherently photodetected and electrically filtered. Signal path delays are pre-compensated on the ground-station site. This system shall be scaled up for a long-range demonstration within the EU-funded H2020-project VERTIGO. In this paper, we investigate the performance and scalability of this uplink configuration. When targeting a capacity of Terabits/s, spectral efficiency must be carefully addressed. Direct detection with optical pre-amplification of OOK or DPSK signals is a conventional optical fiber receiver technique, for which the bandwidth of the optical filter can be kept larger than the signal bandwidth only to a certain extent. Above a certain optical filter bandwidth relative to the signal bandwidth, degradation of the signal-to-noise ratio (SNR) becomes significant because of a stronger ASE $\times$ ASE noise term.

We focus on optimizing power and spectral efficiencies as these are impacted by the introduction of diversity channels. Because these optimizations are essentially independent of the fading channel, we do not assess the reduction of scintillation through channel diversity. We formulate the SNR in terms of number of uplink beams, filter bandwidths (optical and electrical), wavelength channel spacing and received power (in photons per bit). Although the SNR is ideally proportional to the received power, we show how increasing the number of beams while keeping the total received power constant will result in a significant SNR degradation at some point. An optimum for the optical filter bandwidth and for the spacing between the wavelength-division diversity channels is investigated. Polarization-division spatial diversity is kept as an option.
\end{abstract}

Keywords: optical satellite feeder link, multi-aperture, incoherent combining, optical communication, channel diversity, optical preamplifier

\section{INTRODUCTION}

Satellite communication has found its way to applications like high-speed internet, broadcasting, multimedia and data services. Satellites are good candidates to support future connectivity requirements for areas underserved by terrestrial networks and areas where fiber-based networks cannot be deployed. Current existing telecommunication satellites (High Throughput Satellites, HTS) do not support the required throughput for serving wide areas with high-speed internet access. The aggregated throughput easily reaches the Tbit/s range per feeder-link. To serve the next satellite class, known as Very High Throughput Satellites (VHTS), new technological approaches are necessary. Increasing the usable bandwidth and improving the spectral efficiency are two ways to enhance the capacity [1].

Within the VERTIGO project, key technologies are developed for an optical feeder link to a VHTS [2]. The design of freespace optical (FSO) links is impacted by atmospheric impairments. To increase the link robustness with respect to turbulence, adaptive optics can be applied [3]-[5]. An alternative and complementary turbulence mitigation method is the array of apertures (or telescopes) to implement spatial diversity. 
Preliminary measurements with a multi-aperture optical communication setup were performed at Fraunhofer HHI with a four-aperture ground terminal [6]. A bidirectional $20 \mathrm{Gbit} / \mathrm{s}$ OOK link was demonstrated over $400 \mathrm{~m}$ terrestrial test range. In the uplink, each beam emitted by a telescope has a different wavelength to ensure orthogonality and avoid interference between the channels, but all beams carry the same data. In principle, orthogonality between diversity beams can be achieved also with polarization division or phase division [7]. To fulfill the VHTS requirements towards data rates in Tbit/s range, more apertures and wavelengths shall be deployed, which can be used as spatial diversity channels as well as wavelength-division multiplexing (WDM), increasing the total data rate and the stability of the link. This paper analyzes the scalability of spatial-diversity channels for the uplink and its effect on the overall performance. Results shall help dimensioning the optical array and the wavelength channels. We focus on optimizing power and spectral efficiencies as these are impacted by the introduction of diversity channels. Because these optimizations are essentially independent of the fading channel, we do not assess the reduction of scintillation through channel diversity. That is, fluctuations are ignored, only mean received powers are considered.

The paper is structured as follows. In $\S 2$, an analytical model is presented for the signal-to-noise ratio (SNR) of an optical direct-detection receiver with pre-amplification [8]. The SNR formula is extended to multiple diversity channels with a dependence on specific filter bandwidths. In $\S 3$, we introduce the number of photons per bit (PPB) to assess the power efficiency as a function of the number of diversity channels. In $\S 4$, motivated by an optimization of the spectral efficiency, the effect of the spectral spacing between the diversity channels is investigated. Summary and outlook are provided in $\S 5$.

\section{SYSTEM DESCRIPTION AND MODELING}

\subsection{Uplink system setup}

The uplink setup is depicted in Figure 1 for 4 diversity channels in a similar way to [6]. On ground, four continuous-wave lasers of different wavelengths are combined and modulated jointly. After separation and amplification, the four different wavelengths are fed to four optical antennas. All antennas point towards a receiving optical antenna on the satellite. Since the spatially diverse wavelengths do not interfere with each other, the combined all optical power is combined. The respective optical powers are combined in the receive fiber. All antennas are equipped with a mirror-based tracking system (not depicted in Figure 1) which also contributes to scintillation reduction. Optical path lengths are equalized with delay lines.

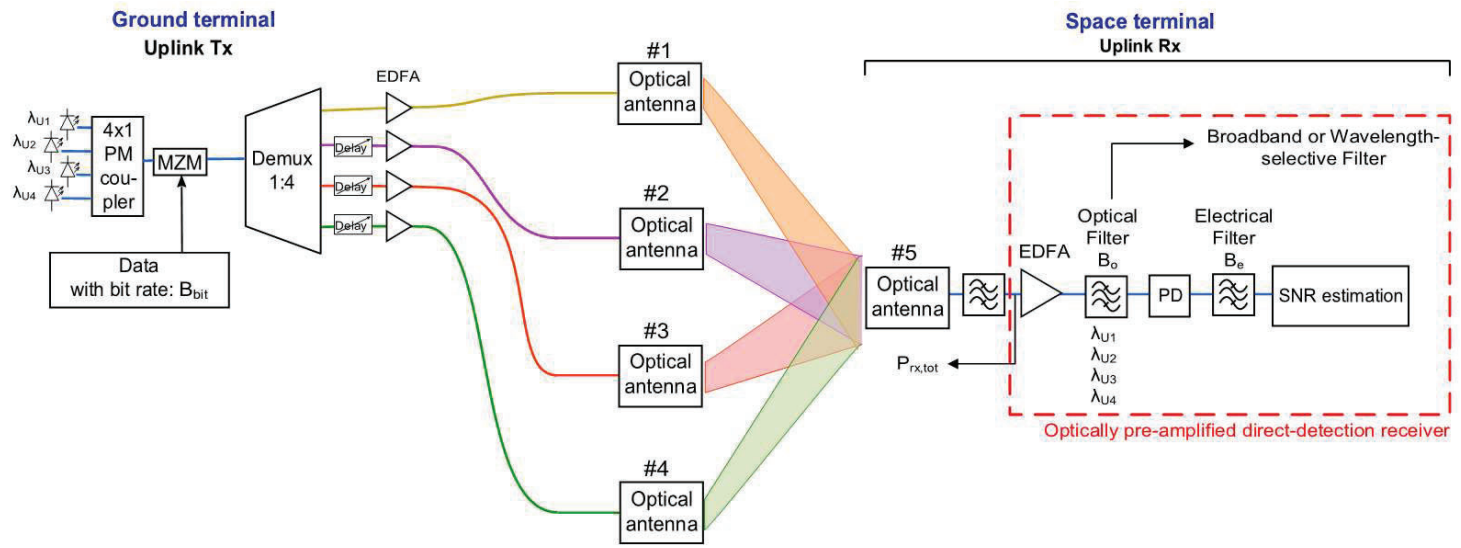

Figure 1: Block diagram of the experimental uplink; Demux: demultiplexer, MZM: Mach-Zehnder modulator, EDFA: Erbium-doped fiber amplifier, PD: photodiode. 


\subsection{Model of optically pre-amplified direct-detection receiver}

The focus of this paper is on the analysis and modeling of the uplink receiver. Incoming beams of the uplink diversity channels are optically combined at the receiver antenna. The receiver model is marked in Figure 1 and consists of an optical amplifier, an optical filter, a photodetector and an electrical filter before data and clock are recovered. In the pre-amplified optical receiver, the amplified spontaneous emission (ASE) noise dominates all other noises [8]. Therefore, we must determine the powers of the ASE noise components to estimate the SNR.

\subsection{SNR for a single wavelength channel}

We revisit the optical-amplifier SNR analysis in [9] and [10]. The power $P_{A S E}$ of the ASE generated by the amplifier over a bandwidth $B_{o}$ is

$$
P_{A S E}=p \cdot n_{s p}(G-1) h v B_{o},
$$

where $n_{s p}$ is the spontaneous emission factor of the EDFA, $p$ is the number of polarizations at its output, $h v$ is the photon energy. The photodetector acts as a square-law operator for the electric fields. The DC photocurrents of signal and noise are

$$
\begin{gathered}
I_{S}=G P_{r x} \eta_{\text {in }} \eta_{\text {out }} \frac{q}{h v}, \\
I_{A S E}=p \cdot n_{s p}(G-1) B_{o} \eta_{\text {out }} q,
\end{gathered}
$$

with $P_{r x}$ the received power, $G$ the amplifier gain, $q$ the elementary charge, $\eta_{\text {in }}$ and $\eta_{\text {out }}$ the losses before and after optical amplification. The photocurrent power consists of three terms: the signal, a mixing term between signal and ASE noise and pure ASE noise. These powers, after electrical filtering with bandwidth $B_{e}$, are

$$
\begin{gathered}
I_{S \times S}^{2}=\left(P_{r x} \eta_{\text {in }} G \eta_{\text {out }} \frac{q}{h v}\right)^{2}, \\
I_{S \times A S E}^{2}=4 n_{s p} \eta_{\text {out }}^{2} G(G-1) P_{r x} \frac{q}{h v} B_{e}, \quad \text { with } B_{e}<\frac{B_{o}}{2}, \\
I_{A S E \times A S E}^{2}=p \cdot\left(n_{\text {sp }} \eta_{\text {out }}(G-1) q\right)^{2} B_{e}\left(2 B_{o}-B_{e}\right), \quad \text { with } B_{e}<B_{o} .
\end{gathered}
$$

With $G>>1$, the SNR formulated as $I_{S \times S}^{2} /\left(I_{S \times A S E}^{2}+I_{A S E \times A S E}^{2}\right)$ is

$$
S N R=\frac{P_{r x}^{2} \eta_{i n}^{2}}{4 n_{s p} \eta_{i n} P_{r x} h v B_{e}+p \cdot n_{s p}^{2}(h v)^{2} B_{e}\left(2 B_{o}-B_{e}\right)} .
$$

\section{POWER EFFICIENCY}

\subsection{SNR analysis for transmitter diversity}

In Eq. (7), the number of diversity channels $n_{c h}$ will influence only the received signal power and the optical filter bandwidth. Assuming $P_{r x}$ and $B_{o}$ are respectively the received power and the optical filter bandwidth of each wavelength channel, the total power and optical filter bandwidth of all channels combined at the receiver can be expressed as

$$
\begin{aligned}
& P_{r x, t o t}=n_{c h} P_{r x}, \\
& B_{o, t o t}=n_{c h} B_{o}, \quad \text { with } B_{o} \leq B_{C h s p},
\end{aligned}
$$

where $B_{C h s p}$ is the diversity channel spacing as illustrated in Figure 2. The case $B_{o}=B_{C h s p}$ would correspond to a single broadband filter covering all channels whereas the case $B_{o}<B_{C h s p}$ is associated to a wavelength-selective filter. 


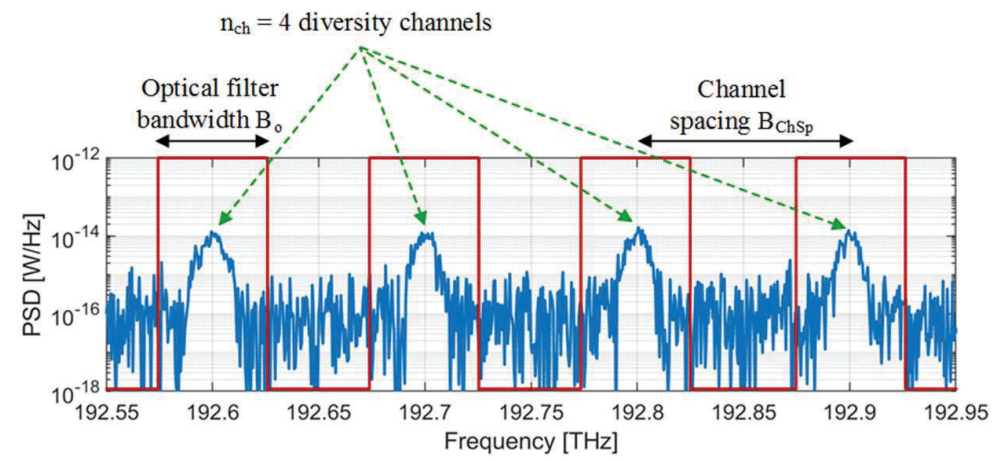

Figure 2: Power spectral density (PSD) of a simulated received signal after optical amplification. Four beams with $100-\mathrm{GHz}$ spacing carry the same $10 \mathrm{Gbit} / \mathrm{s}$ signal. The bandwidth of the optical wavelength-selective filter is marked.
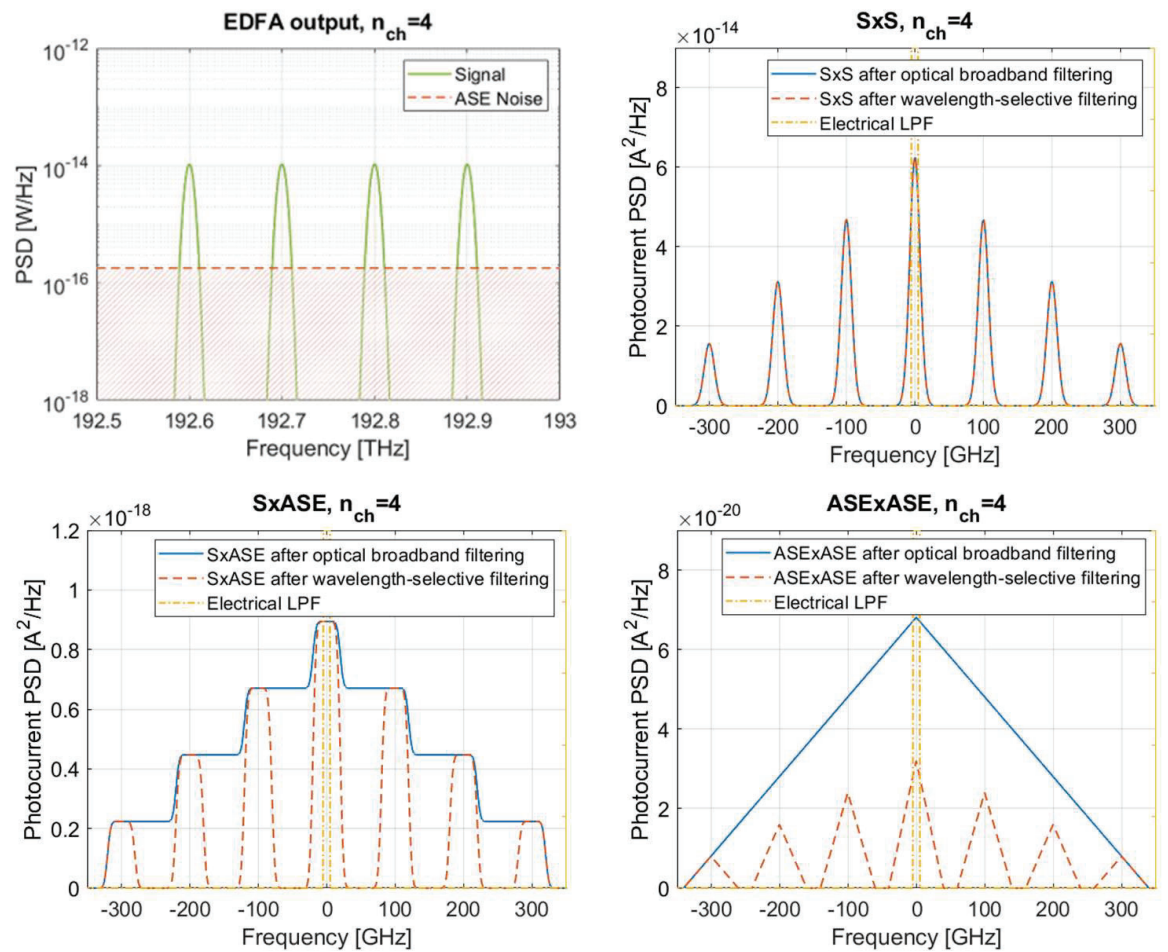

Figure 3: Spectrums of signal and noise before and after photodetection for four beams $\left(n_{c h}=4\right)$ with $100-\mathrm{GHz}$ wavelength spacing.

To visualize the impact of $n_{c h}$ on Eqs. (4) to (6), the spectrums of the signal and noise terms are plotted in Figure 2 for four carriers, modelled here as Gaussian functions. The transfer function of the electrical low-pass filter is also depicted. The electrical signal spectrum exhibits a central peak and $2\left(n_{c h}-1\right)$ beat signals or side lobes resulting from the convolution of the optical spectrum. Only the central peak is kept after electrical filtering. The amplified signal and noise components (after optical broadband filtering) are illustrated with the blue lines. Eqs (5) and (6) show that only the S $\times$ ASE term depends on parameter $B_{o}$ and in turn $n_{c h}$. Figure 3 illustrates the suppression of the beat components (or side-lobes) of the $\mathrm{S} \times \mathrm{ASE}$ convolution by using an electrical filter after the photodetection with $B_{e} \ll B_{o}$. For the ASE $\times$ ASE term 
however, the height of the triangular spectrum is directly proportional to $B_{o, t o t}$ and affects the SNR as $n_{c h}$ increases, even after electrical filtering. This contribution can be reduced by using a wavelength-selective optical filter.

Substituting the parameters $P_{r x}$ and $B_{o}$ with, respectively, $P_{r x, t o t}$ and $B_{o, \text { tot }}$ in Eq (7) and setting $\eta_{\text {in }} \approx 1$, the SNR of a pre-amplified optical receiver with transmitter diversity becomes

$$
S N R=\left(\frac{1}{4 n_{s p} k_{e}}\right) \frac{n_{c h} n_{P P B . P A}^{2}}{n_{P P B, P A}+n_{s p} k_{o}},
$$

where $n_{P P B, P A}=P_{r x} /\left(h v B_{b i t}\right)$ is the number of PPB per aperture, $B_{b i t}$ is the bit rate, $k_{e} \equiv B_{e} / B_{b i t}$ and $k_{o} \equiv B_{o} / B_{b i t}$. Eq. (10) gives an SNR proportional to the number $n_{c h}$ of wavelength-division diversity (WDD) channels, i.e. the number of apertures. So, the size of the telescope array is here used to scale the transmit power and thus increases the SNR of the link. For the case that ASE $\times$ ASE is negligible, the SNR of Eq. (10) simplifies to $\left(1 / 4 n_{s p} k_{e}\right) n_{c h} n_{P P B, P A}$ which is proportional to the number of PPB.

Now, if we want to assess the total power efficiency, we shall consider the total number of PPB that is required to reach a satisfactory SNR instead of considering the number of PPB per aperture $\left(n_{P P B, P A}\right)$. Therefore, by setting

$$
n_{P P B} \equiv n_{c h} n_{P P B, P A},
$$

Eq. (10) yields

$$
S N R=\left(\frac{1}{4 n_{s p} k_{e}}\right) \frac{n_{P P B}^{2}}{n_{P P B}+n_{c h} n_{s p} k_{o}}
$$

In this formulation, if ASE $\times$ ASE noise is negligible, the SNR stays constant for different $n_{c h}$. If ASE $\times$ ASE noise dominates, the SNR decreases by increasing $n_{c h}$.
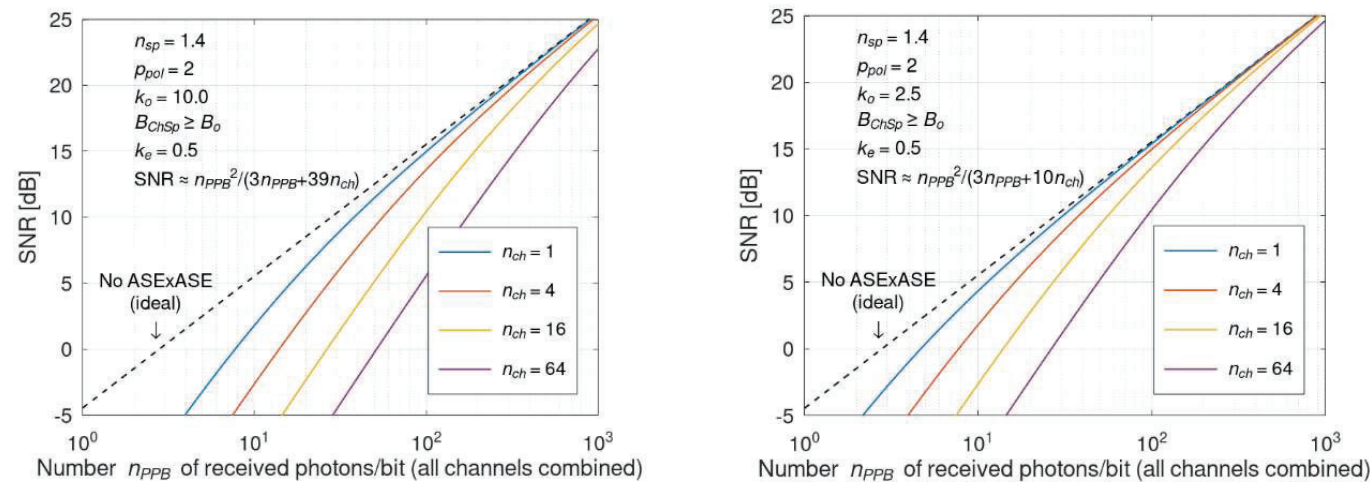

Figure 4: SNR as a function of the total number of received PPB. Power efficiency decreases with more diversity channels due to the increased ASE noise. Left: optical filter bandwidth parameter is set to $k_{o}=B_{o} / B_{b i t}=10$. Right: same as left graph but with optical filter bandwidth parameter $k_{o}=2.5$.

The SNR performance of Eq. (12) is plotted in Figure 4 as a function of $n_{P P B}$ and $n_{c h}$ compared to the ideal case with no ASE $\times$ ASE. The goal is to keep the required received power as low as possible while increasing the number of channels. The plot shows that as $n_{P P B}$ decreases below the ASE $\times$ ASE noise level $n_{c h} n_{s p} k_{o}$, the SNR deviates from the ideal case. In the two graphs of Figure 4 the optical filter bandwidth per channel is set respectively to $B_{o}=10 B_{b i t}$ and $B_{o}=2.5 B_{b i t}$.

With $k_{o}=10$, the left graph of Figure 4 corresponds for example to a 10-Gbit/s signal with a 100-GHz spacing and a broadband optical filtering. For a targeted SNR of $10 \mathrm{~dB}$ with $k_{o}=10$, about $40 \mathrm{PPB}$ is needed for $n_{c h}=1$ beam and the chosen model parameters. Increasing the number of channels to $n_{c h}=64$ while keeping the total power constant $\left(n_{P P B}=\right.$ 40) leads to a SNR degradation of $-12 \mathrm{~dB}$. In this case, the total power must be increased to 180 PPB (i.e., increase of 
$+6.5 \mathrm{~dB}$ ) to achieve again an SNR of $10 \mathrm{~dB}$. When we divide the optical noise bandwidth by four and set $k_{o}=2.5$ for the right graph, about $30 \mathrm{PPB}$ is needed to reach an SNR of $10 \mathrm{~dB}$ for $n_{c h}=1$ beam. The total power must be increased to 90 PPB (i.e., increase of $+5 \mathrm{~dB}$ ) to achieve again an SNR of $10 \mathrm{~dB}$.

\section{SPECTRAL EFFICIENCY}

An additional critical criterion for VHTS feeder link system design (besides power efficiency) is the spectral efficiency of the link. As the number of WDD channels increases for the link, the utilized spectrum becomes broader. As this spectrum is occupied for spatial diversity and not for data transmission, spectral efficiency decreases. The diversity channels in the setup of [6] have a $100 \mathrm{GHz}$ channel spacing. One way to enhance the spectral efficiency is to reduce the wavelength spacing. Although, in practice, the choice of the wavelength spacing may be subject to the availability of the WDM components, we here analyze arbitrary channel spacing values and evaluate the possible interferences at the receiver (see Figure 5). Impairments caused by the overlap of WDD channels differ from the WDM crosstalk because, unlike multiplexing channels, diversity channels carry the same data.

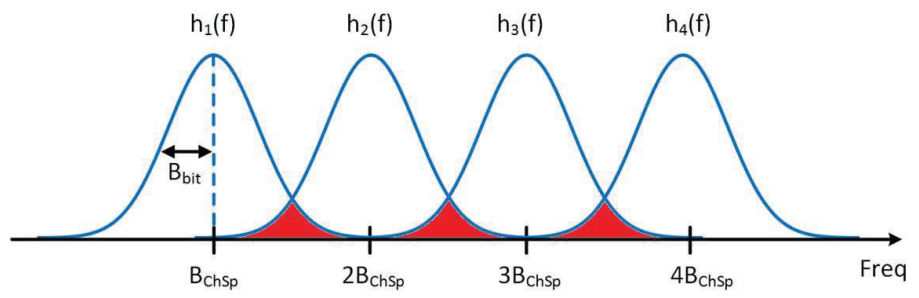

Figure 5. Spectral overlap of diversity channels due to a small channel spacing.

Let $h\left(f+m B_{C h s p}\right)$ be the amplitude spectrum of the $m^{\text {th }}$ diversity channel where $B_{C h s p}$ is again the channel spacing. To obtain the total power $P_{r x, \text { tot }}$ of the combined channels, we must consider the phase $\varphi_{m}$ of each channel before adding the channels and integrating their power densities. Doing so, we have

$$
P_{r x, t o t}=\int\left|\sum_{m=1}^{n_{c h}} h\left(f+m B_{C h s p}\right) e^{i \varphi_{m}}\right|^{2} d f .
$$

We consider two extreme cases where adjacent channels have a phase difference of either 0 or $\pi$ :

$$
\begin{cases}\varphi_{m+1}-\varphi_{m}=0, & \text { (best case) }, \\ \varphi_{m+1}-\varphi_{m}=\pi, & \text { (worst case). }\end{cases}
$$

Introducing the new values of $P_{r x, t o t}$ from Eq. (13) into Eq. (12) and considering Gaussian-shaped power densities, the SNR can be evaluated as a function of the channel spacing parameter $k_{C h s p}=B_{C h s p} / B_{b i t}$. This is done in Figure 6 for the case $n_{P P B}=100$ under no inter-channel interference. The data rate $B_{b i t}$ has been set to the full width at half maximum (FWHM) of the Gaussian power densities. In the best case, the beams interfere constructively and the system is operated as a phased array. The ideal case of an optical phased array emitting at a single wavelength leads to $P_{r x, t o t}=P_{r x} n_{c h}^{2}$ which can be seen in Figure 6 from the high SNR values at $k_{C h s p}=0$. The setting $k_{o}=\min \left(5, k_{C h s p}\right)$ in Figure 6 means that the optical filter bandwidth $B_{o}$ equals the channel spacing $B_{C h s p}$ as long as $B_{C h s p}<5 B_{b i t}$, and that $B_{o}$ is kept constant at a value of $5 B_{b i t}$ for higher $B_{C h s p}$. This explains the flat curves on the right part of Figure 6 . Note that if a broadband filter was used, the SNR performance would decrease with increasing channel spacing, since more spectral spacing between the channels translates into more ASE noise. Furthermore, the upper and lower limits of the SNR based on Eq. (13) are depicted. The SNR ranges between these two limits depending on the random phase differences between the diversity channels. This model simplifies to a great extent the real process. For example, inter-channel destructive interferences can also introduce inter-symbol interference (ISI) due to the decreased bandwidth. Additionally, the turbulence-induced power fluctuations, which are ignored here, will modify the strength of the interferences. However the presented results indicate that for a reliable communication a WDD channel spacing with $k_{C h s p}>2$ must be guaranteed, with $k_{C h S p}=B_{C h s p} / B_{b i t}$. 
In particular, the channel spacing can be reduced to e.g. $25 \mathrm{GHz}$ for $10 \mathrm{Gbit} / \mathrm{s}$ or $50 \mathrm{GHz}$ for $20 \mathrm{Gbit} / \mathrm{s}$, leading to two times better spectral efficiency compared to [6].

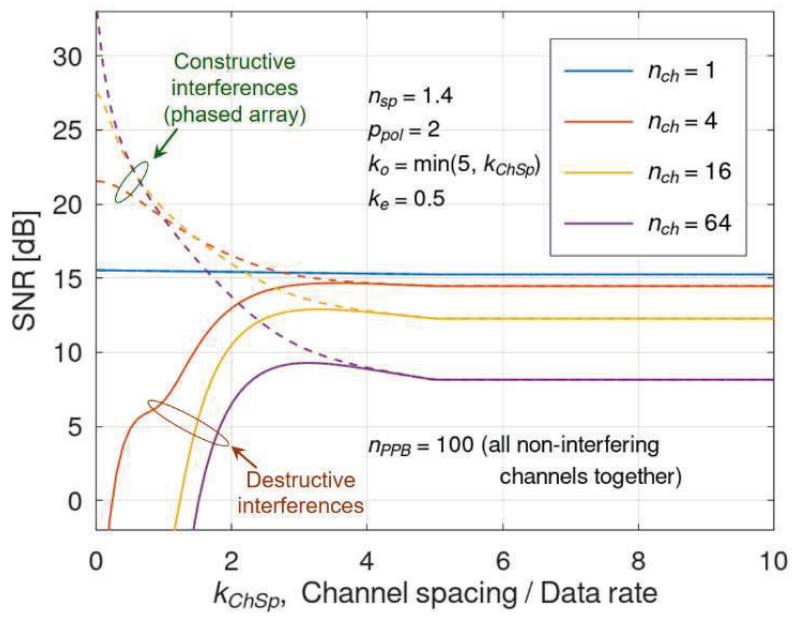

Figure 6. SNR vs. channel spacing for different number $n_{c h}$ of WDD channels. At low channel spacing, a worst case is given by destructive interferences between adjacent channels. Constructive interferences (dashed lines) provide the best case.

\section{CONCLUSIONS}

In the context of an optical satellite uplink with direct detection and optical pre-amplification, we have described how the implementation of a spatial-diversity scheme impacts the power and spectral efficiencies. With many wavelength-division diversity channels, ASE noise worsens the power efficiency. Optical filter bandwidth must be minimized to reduce the ASE $\times$ ASE term. When looking for a minimum spectral spacing between the spatial-diversity wavelength channels, we obtain significant interference-induced losses for a channel spacing less than twice the data rate

Prospectively, one way to improve the spectral efficiency by a factor 2 would be to implement spatial polarization-division diversity in addition to spatial WDD. Preliminary outdoor measurements of orthogonal polarizations at Fraunhofer HHI have given promising results in this regard. Additionally, one way to improve power efficiency would be an upgrade to coherent detection where the ASE $\times$ ASE term would be suppressed. This option has a much higher complexity as we would need one coherent receiver per diversity channel. But previous works have shown the benefits of this configuration [11]. Because WDD may conflict with WDM regarding the needed spectrum, one may ultimately implement an adaptive modeswitching between WDM and WDD so that spectrum resources are free from diversity to multiplexing whenever turbulence becomes weaker.

\section{ACKNOWLEDGEMENTS}

This work is part of the VERTIGO project. VERTIGO has received funding from the European Union's Horizon 2020 research and innovation program under Grant Agreement No. 822030.

\section{REFERENCES}

[1] O. Kodheli et al., "Satellite Communications in the New Space Era: A Survey and Future Challenges," in IEEE Communications Surveys \& Tutorials 
[2] A. Le Kernec et al., "The H2020 VERTIGO Project Towards Tbit/s Optical Feeder Links", ICSO Conference (2020)

[3] N. Védrenne et al., "First Experimental Demonstration of Adaptive Optics Pre-Compensation for GEO Feeder Links in a Relevant Environment", IEEE International Conference on Space Optical Systems and Applications (ICSOS), Oct 2019, Portland

[4] R. Saathof et al., "Optical technologies for terabit/s-throughput feeder link," 2017 IEEE International Conference on Space Optical Systems and Applications (ICSOS), Naha, 2017, pp. 123-129

[5] A. Brady et al., "Demonstrated pre-compensation of a focused laser beam with up to $0.27 \mathrm{mrad}$ point-ahead-angle over a 1-km horizontal communication path," Proc. SPIE 11180, International Conference on Space Optics ICSO 2018, 111801E (12 July 2019)

[6] N. Perlot, P. Hanne and J. Perdigues, "Bidirectional 20-Gbit/s OOK Link with a 4-Telescope Array and Incoherent Signal Combining," IEEE International Conference on Space Optical Systems and Applications (ICSOS), Portland, OR, USA, 2019

[7] C. Fuchs, D. Giggenbach, R. M. Calvo and W. Rosenkranz, "Transmitter Diversity with Phase-Division for Optical GEO Feeder Links," Photonic Networks, 20th ITG-Symposium, Leipzig, Germany, 2019

[8] A. Maho, M. Faugeron, A. Le Kernec, K. Elayoubi, M. Sotom, "Assessment of the effective performance of DPSK vs. OOK in satellite-based optical communications," Proc. SPIE 11180, International Conference on Space Optics - ICSO 2018

[9] S. B. Alexander, Optical Communication Receiver Design, volume 22 of TT22. SPIE, Bellingham, 2006

[10] N. A. Olsson, "Lightwave systems with optical amplifiers," in Journal of Lightwave Technology, vol. 7, no. 7, pp. 1071-1082, July 1989

[11]D. J. Geisler et al. "Multi-aperture digital coherent combining for free-space optical communication receivers". Opt Express 24, 12661-12671 (2016) 